\title{
Optimization of Statistical Decisions for Age Replacement Problems via a New Pivotal Quantity Averaging Approach
}

\author{
Nicholas A. Nechval ${ }^{1,}$, , Gundars Berzins ${ }^{2}$, Vadims Danovics ${ }^{3}$ \\ ${ }^{1}$ Department of Mathematics, Baltic International Academy, Riga, Latvia \\ ${ }^{2}$ Department of Management, University of Latvia, Riga, Latvia \\ ${ }^{3}$ Department of Marketing, University of Latvia, Riga, Latvia \\ Email address: \\ nechval@junik.lv (N. A. Nechval), gundars.berzins@lu.lv (G. Berzins), vadims.danovics@lu.lv (V. Danovics)
}

\section{To cite this article:}

Nicholas A. Nechval, Gundars Berzins, Vadims Danovics. Optimization of Statistical Decisions for Age Replacement Problems via a New Pivotal Quantity Averaging Approach. American Journal of Theoretical and Applied Statistics. Special Issue: Novel Ideas for Efficient Optimization of Statistical Decisions and Predictive Inferences under Parametric Uncertainty of Underlying Models with Applications. Vol. 5, No. 2-1, 2016, pp. 21-28. doi: 10.11648/j.ajtas.s.2016050201.14

\begin{abstract}
Age replacement strategies, where a unit is replaced upon failure or on reaching a predetermined age, whichever occurs first, provide simple and intuitively attractive replacement guidelines for technical units. Within theory of stochastic processes, the optimal preventive replacement age, in the sense of leading to minimal expected costs per unit of time when the strategy is used for a sequence of similar units over a long period of time, is derived by application of the renewal reward theorem. The mathematical solution to the problem of what is the optimal age for replacement is well known for the case when the parameter values of the underlying lifetime distributions are known with certainty. In actual practice, such is simply not the case. When these models are applied to solve real-world problems, the parameters are estimated and then treated as if they were the true values. The risk associated with using estimates rather than the true parameters is called estimation risk and is often ignored. When data are limited and (or) unreliable, estimation risk may be significant, and failure to incorporate it into the model design may lead to serious errors. Its explicit consideration is important since decision rules that are optimal in the absence of uncertainty need not even be approximately optimal in the presence of such uncertainty. In the present paper, for efficient optimization of statistical decisions under parametric uncertainty, the pivotal quantity averaging (PQA) approach is suggested. This approach represents a new simple and computationally attractive statistical technique based on the constructive use of the invariance principle in mathematical statistics. It allows one to carry out the transition from the original problem to the equivalent transformed problem (in terms of pivotal quantities and ancillary factors) via invariant embedding a sample statistic in the original problem. In this case, the statistical optimization of the equivalent transformed problem is carried out via ancillary factors. Unlike the Bayesian approach, the proposed approach is independent of the choice of priors. This approach allows one to eliminate unknown parameters from the problem and to find the better decision rules, which have smaller risk than any of the well-known decision rules. To illustrate the proposed approach, the numerical examples are given.
\end{abstract}

Keywords: Age Replacement, Parametric Uncertainty, Equivalent Transformed Problem, Optimization

\section{Introduction}

Attention to age replacement has predominantly been based on a classical operational research (OR) perspective, where the probability distribution for the lifetime of the unit is assumed to be known. Age replacement strategies for technical units describe that a unit is replaced preventively upon reaching age $T$, or correctively upon failure before $T$, where preventive replacements are typically less expensive than corrective replacements. The classical mathematical approach for determining the optimal replacement age is based on the renewal criterion, which implicitly assumes that the same replacement strategy is used over a very long period of time, consisting of many cycles, where one cycle is the period of random length between two consecutive replacements; see, for example, Barlow and Hunter [1], Barlow and Proschan $[2,3]$. They discussed the problem of determining an optimal preventive replacement age $T$ to minimize the long-run average expected cost per unit time over the infinite horizon (the average cost in short). Since then, this basic model has been generalized and modified by many 
authors to handle more practical situations, as summarized in Ascher and Feingold [4], Nakagawa [5], and Valdez-Flores and Feldman [6]. In practice, this procedure is also used even though one realizes that the resulting optimal strategy may only be used for a few such cycles, for example, because the unit would normally undergo some technical updates within reasonable period of time, or one wishes to keep the option open to change the policy in light of new information that may occur during the process.

It should be noted that Barlow and Hunter [1] considered only minimal repair and preventive replacement as maintenance activities. Minimal-repair and replacement are often used as practical maintenance activities of real reliability systems. A minimal repair is the maintenance activity to repair the failed system so that its function is recovered, without changing its age, while a replacement restores the entire system into the new condition so that it behaves as a new system. Further, replacement is classified into preventive replacement or failure (or corrective) replacement according as whether the system is in operation or in failure. Phelps [7] introduced failure replacement as a maintenance activity, and discussed an optimal maintenance problem with minimal repair and failure replacement under the average cost criterion (since it was assumed in this model that the required costs for preventive and failure replacements are equal, the system should be replaced only when it is failed). Tahara and Nishida [8] discussed the maintenance problem with both preventive replacement and failure replacement which have different costs. The above studies concern, in general, the classical age replacement model and its some modifications.

\section{Classical Age Replacement Model}

In the well-known classical model for age replacement (Barlow and Proschan [2, 3]), the failure time of the unit (component) is assumed to be an absolutely continuously distributed random variable $X \geq 0$ with known probability distribution, with cumulative distribution function (cdf) $F(x)=$ $\operatorname{Pr}(X \leq x)$, probability density function (pdf) $f(x)$, hazard rate $h(x)=f(x) /(1-F(x))$, and expected value $E(X)$. It is assumed that $h(x)$ is monotonously strictly increasing, which is often considered to be a natural assumption for situations where age replacement may be cost effective (Barlow and Proschan [2, 3]) To avoid mathematical complications, it is assumed that $F(0)=0, F(x)>0$ for all $x>0$, and $E(X)<\infty$.

The simple age replacement policy of replacing a component when it fails or at time $T$, whichever comes first, requires fixed costs $c_{1}$ and $c_{2}$ for replacing a failed and non-failed item, respectively, with $c_{1}>c_{2}>0$. Let $N_{1}(t)$ and $N_{2}(t)$ denote the number of failed and non-failed units, respectively, which are replaced in $(0, t]$. The optimum age replacement interval, $T^{*}$, is chosen so as to minimize the limiting expected cost per unit time, $C(T)$ (Barlow and Proschan [2], Glasser [9], Scheaffer [10]), where

$$
\begin{aligned}
& C(T)=\lim _{t \rightarrow \infty} E\left\{c_{1} N_{1}(t)+c_{2} N_{2}(t)\right\} / t \\
&=\frac{c_{1}-\left(c_{1}-c_{2}\right) \bar{F}(T)}{T}, \\
& \int_{0}^{T} \bar{F}(x) d x
\end{aligned}
$$

$$
\bar{F}(x)=1-F(x)
$$

It can be shown (Berg [11]) that if $h(T)$ increases in $T$ and if,

$$
\lim _{T \rightarrow \infty} h(T)>\frac{c_{1}}{\left(c_{1}-c_{2}\right) E\{X\}},
$$

then there exists a unique minimum of (1) and $T^{*}$ is the unique solution of the optimality equation

$$
h(T) \int_{0}^{T} \bar{F}(x) d x+\bar{F}(T)=\frac{c_{1}}{c_{1}-c_{2}} .
$$

Conditions for the existence of a unique minimum are established and an equation characterizing the optimal policy and minimal average costs is derived.

In this paper, for efficient optimization of statistical decisions (based on a past random sample of lifetimes) under parametric uncertainty, the pivotal quantity averaging (PQA) approach is suggested. This approach represents a simple and computationally attractive statistical technique based on the constructive use of the invariance principle in mathematical statistics. It allows one to carry out the transition from the original problem to the equivalent transformed problem (in terms of pivotal quantities and ancillary factors) via invariant embedding a sample statistic in the original problem (Nechval and Vasermanis [12]). In this case, the statistical optimization of the equivalent transformed problem is carried out via ancillary factors. Unlike the Bayesian approach, the proposed approach is independent of the choice of priors. It allows one to eliminate unknown parameters from the problem and to find the better decision rules, which have smaller risk than any of the well-known decision rules.

\section{Innovative Age Replacement Model}

In this paper, to handle more practical situations, an innovative model for age replacement is proposed. The costs included in the age replacement model are not assumed to be constants.

Consider a unit (component) with a stochastic lifetime $X$ with $\operatorname{cdf} F_{\theta}(x)$ and $\operatorname{pdf} f_{\theta}(x)$, where $\theta$ is a parameter (in general, vector). It is assumed that $f_{\theta}(x)$ is continuous in $x$. If one adopts a policy of replacing the unit either at failure ('corrective replacement' at cost $c_{1}(\tau-X)$ ), or at some specified replacement age $\tau$ ('preventive replacement' at cost $c_{2}(X-\tau)>$ $0)$, whichever occurs first, the cost pet unit is 


$$
c(\tau)=\left\{\begin{array}{l}
c_{1}(\tau-X) \text { if } X<\tau, \\
c_{2}(X-\tau) \text { if } X \geq \tau,
\end{array}\right.
$$

where $c_{1}$ and $c_{2}$ are assumed to be known constants with $c_{1}>$ $c_{2}>0$, a logical requirement to make preventive replacement possibly worthwhile; while the usage per unit is

$$
t(\tau)=\min (X, \tau)
$$

The expected cost per unit is

$$
\begin{gathered}
C_{\theta}(\tau)=E_{\theta}\{c(\tau)\} \\
=c_{1} \int_{0}^{\tau}(\tau-x) f_{\theta}(x) d x+c_{2} \int_{\tau}^{\infty}(x-\tau) f_{\theta}(x) d x \\
=c_{1} \tau+c_{2} E_{\theta}\{X\}-\left(c_{1}+c_{2}\right) \int_{0}^{\tau} \bar{F}_{\theta}(x) d x,
\end{gathered}
$$

while the expected usage per unit is

$$
T_{\theta}(\tau)=\int_{0}^{\tau} x f_{\theta}(x) d x+\tau \int_{\tau}^{\infty} f_{\theta}(x) d x=\int_{0}^{\tau} \bar{F}_{\theta}(x) d x .
$$

The performance index (objective function) of the innovative age replacement model is given by

$$
\begin{aligned}
\Lambda_{\theta}(\tau)=\frac{C_{\theta}(\tau)}{T_{\theta}(\tau)}=\frac{c_{1} \tau+c_{2} E_{\theta}\{X\}-\left(c_{1}+c_{2}\right) \int_{0}^{\tau} \bar{F}_{\theta}(x) d x}{\int_{0}^{\tau} \bar{F}_{\theta}(x) d x} \\
=\frac{c_{1} \tau+c_{2} E_{\theta}\{X\}}{\int_{0}^{\tau} \bar{F}_{\theta}(x) d x}-\left(c_{1}+c_{2}\right) .
\end{aligned}
$$

It measures the effectiveness of a replacement policy over an unlimited time span, because it represents the long-run average cost per unit of time of utilization.

Remark 1. If

$$
c(\tau)=\left\{\begin{array}{l}
c_{1} \text { if } X<\tau, \\
c_{2} \text { if } X \geq \tau
\end{array}\right.
$$

then the performance index of the classical age replacement model takes place:

$$
\Lambda_{\theta}(\tau)=\frac{c_{1}-\left(c_{1}-c_{2}\right) \bar{F}_{\theta}(\tau)}{\int_{0}^{\tau} \bar{F}_{\theta}(x) d x} .
$$

\section{Optimization of Replacement Policy Under Complete Information}

\subsection{Optimality Equation}

To obtain the optimality equation for $\tau$, the performance index (9) will be differentiated with respect to $\tau$ and set equal to zero to provide the optimal value of $\tau$ as follows:

$$
\frac{d \Lambda_{\theta}(\tau)}{d \tau}=\frac{c_{1} \bar{F}_{\theta}(\tau)\left[\int_{0}^{\tau} \bar{F}_{\theta}(x) d x / \bar{F}_{\theta}(\tau)-\frac{c_{2}}{c_{1}} E_{\theta}\{X\}-\tau\right]}{\left[\int_{0}^{\tau} \bar{F}_{\theta}(x) d x\right]^{2}} .
$$

By requiring this equation to be zero, the desired optimality equation for $\tau$ is obtained as

$$
\tau=\int_{0}^{\tau} \bar{F}_{\theta}(x) d x / \bar{F}_{\theta}(\tau)-\frac{c_{2}}{c_{1}} E_{\theta}\{X\} .
$$

This equation represents a necessary condition for the existence of an optimal solution,

$$
\tau^{*}=\arg \left(\tau=\int_{0}^{\tau} \bar{F}_{\theta}(x) d x / \bar{F}_{\theta}(\tau)-\frac{c_{2}}{c_{1}} E_{\theta}\{X\}\right)
$$

If

$$
E_{\theta}\{X\}=\int_{0}^{\infty} \bar{F}_{\theta}(x) d x<\infty
$$

it follows from (13) that the optimal solution (14) exists and is unique.

To make sure that the optimal solution (14) represents a minimum of (9), not a maximum, the second derivative of the performance index (9) is calculated and evaluated at $\tau=\tau^{*}$ :

$$
\frac{d^{2} \Lambda_{\theta}(\tau)}{d \tau^{2}}=\frac{\left[c_{1} \tau+c_{2} E_{\theta}\{X\}\right] f_{\theta}(\tau)}{\left[\int_{0}^{\tau} \bar{F}_{\theta}(x) d x\right]^{2}}>0
$$

which is positive (i. e., (14) represents a minimum point of (9)), as desired. Thus, the following theorem has been proven.

Theorem 1. Consider units (components) with a stochastic lifetime $X$ with $\operatorname{cdf} F_{\theta}(x)$ and $\operatorname{pdf} f_{\theta}(x)$, where $\theta$ is a parameter (in general, vector). It is assumed that $f_{\theta}(x)$ is continuous in $x$. Then the optimal preventive replacement age,

$$
\tau^{*}=\arg \min _{\tau}\left[\Lambda_{\theta}(\tau)=\frac{C_{\theta}(\tau)}{T_{\theta}(\tau)}\right]
$$




$$
\begin{aligned}
& =\arg \min _{\tau}\left[\frac{c_{1} \tau+c_{2} E_{\theta}\{X\}-\left(c_{1}+c_{2}\right) \int_{0}^{\tau} \bar{F}_{\theta}(x) d x}{\tau}\right] \\
& =\arg \min _{\tau}\left[\left(c_{1} \tau+c_{2} E_{\theta}\{X\}\right) / \int_{0}^{\tau} \bar{F}_{\theta}(x) d x-\left(c_{1}+c_{2}\right)\right] \\
& =\arg \left(\tau=\left(\bar{F}_{\theta}(\tau)\right)^{-1} \int_{0}^{\tau} \bar{F}_{\theta}(x) d x-\frac{c_{2}}{c_{1}} E_{\theta}\{X\}\right),
\end{aligned}
$$

representing a minimum point of the performance index $\Lambda_{\theta}(\tau)$ of the innovative age replacement problem, exists and is unique if and only if $E_{\theta}\{X\}<\infty$.

\subsection{Numerical Example 1}

Consider units with a stochastic lifetime $X$ with

$$
\begin{gathered}
X \sim \operatorname{EXP}(\theta=(\mu, \sigma)), \\
F_{\theta}(x)=1-\exp \left(-\frac{x-\mu}{\sigma}\right), \\
f_{\theta}(x)=\frac{1}{\sigma} \exp \left(-\frac{x-\mu}{\sigma}\right), \\
h_{\theta}(x)=\frac{f_{\theta}(x)}{1-F_{\theta}(x)}=\frac{1}{\sigma}, \quad x>\mu, \\
E_{\theta}\{X\}=\mu+\sigma, \quad \operatorname{var}\{X\}=\sigma^{2},
\end{gathered}
$$

where $\sigma>0$ is a scale parameter and $\mu$ is both a location and a threshold parameter. When $\mu=0$ one gets the well-known one-parameter exponential distribution. The aim is to determine an optimal preventive replacement age $\tau^{*}$. Let us assume that

$$
\mu=0, \sigma=2000, c_{1}=1000, c_{2}=100 .
$$

It follows from (14) that the optimal preventive replacement age is given by

$$
\begin{aligned}
\tau^{*}= & \arg \left(\tau=\int_{0}^{\tau} \bar{F}_{\theta}(x) d x / \bar{F}_{\theta}(\tau)-\frac{c_{2}}{c_{1}} E_{\theta}\{X\}\right) \\
& =\arg \left(\tau=\sigma\left[\exp \left(\frac{\tau}{\sigma}\right)-\left(1+\frac{c_{2}}{c_{1}}\right)\right]\right) \\
& =832.4423
\end{aligned}
$$

It follows from (9) that the minimal value of the performance index is

$$
\begin{gathered}
\Lambda_{\theta}\left(\tau^{*}\right)=\frac{c_{1} \tau^{*}+c_{2} E_{\theta}\{X\}}{\tau^{*} \bar{F}_{\theta}(x) d x}-\left(c_{1}+c_{2}\right) \\
\int_{0} \\
=\frac{c_{1} \tau^{*} / \sigma+c_{2}}{1-\exp \left(-\tau^{*} / \sigma\right)}-\left(c_{1}+c_{2}\right)=416.2212 .
\end{gathered}
$$

\subsection{Numerical Example 1 (Continued)}

If

$$
\mu=100, \sigma=2000, c_{1}=1000, c_{2}=100,
$$

then it follows from (14) that the optimal replacement age is given by

$$
\begin{aligned}
& \tau^{*}=\arg \left(\tau=\int_{\mu}^{\tau} \bar{F}_{\theta}(x) d x / \bar{F}_{\theta}(\tau)-\frac{c_{2}}{c_{1}} E_{\theta}\{X\}\right) \\
& =\arg \left(\tau=\sigma\left[\exp \left(\frac{\tau-\mu}{\sigma}\right)-1-\frac{c_{2}}{c_{1}}\left(1+\frac{\mu}{\sigma}\right)\right]\right) \\
& =1119.124 .
\end{aligned}
$$

It follows from (9) that the minimal value of the performance index is equal to

$$
\begin{gathered}
\Lambda_{\theta}\left(\tau^{*}\right)=\frac{c_{1} \tau^{*}+c_{2} E_{\theta}\{X\}}{\tau^{*} \bar{F}_{\theta}(x) d x}-\left(c_{1}+c_{2}\right) \\
=\frac{c_{1} \frac{\tau^{*}}{\sigma}+c_{2}\left(\frac{\mu}{\sigma}+1\right)}{1-\exp \left(-\frac{\tau-\mu}{\sigma}\right)}-\left(c_{1}+c_{2}\right)=564.5621 .
\end{gathered}
$$

\section{Optimization of Replacement Policy Under Parametric Uncertainty}

\subsection{Scale Family of Lifetime Distributions}

Consider a situation described by a scale family of probability distribution functions, indexed by a parameter $\sigma$,

$$
\begin{gathered}
F_{\sigma}(x)=\operatorname{Pr}(X \leq x)=F\left(\frac{x}{\sigma}\right)=F(z), \\
0<x<\infty
\end{gathered}
$$

where $\sigma>0$ is a scale parameter, the distribution of $Z=X / \sigma$ does not depend on any unknown parameter. Let $X_{1}, X_{2}, \ldots, X_{n}$ denote a random sample of component lifetimes, each possessing a distribution function (29) such that $F(0)=0$ and 
$E_{\sigma}\{X\}$ is finite. It is assumed that the scale parameter is unknown, but there exists some statistic $S=S\left(X_{1}, X_{2}, \ldots, X_{n}\right)$ such that,

$$
V=\frac{S}{\sigma}
$$

represents a pivotal quantity whose distribution does not depend on any unknown parameter. In this case, the expected cost per unit (7) and the expected usage per unit (8) are transformed, respectively, as follows:

$$
\begin{gathered}
C_{\sigma}(\tau)=c_{1} \tau+c_{2} \int_{0}^{\infty} \bar{F}\left(\frac{x}{\sigma}\right) d x-\left(c_{1}+c_{2}\right) \int_{0}^{\tau} \bar{F}\left(\frac{x}{\sigma}\right) d x \\
=\sigma\left[c_{1} \frac{\tau}{\sigma}+c_{2} \int_{0}^{\infty} \bar{F}\left(\frac{x}{\sigma}\right) d\left(\frac{x}{\sigma}\right)-\left(c_{1}+c_{2}\right) \int_{0}^{\tau} \bar{F}\left(\frac{x}{\sigma}\right) d\left(\frac{x}{\sigma}\right)\right] \\
=\sigma\left[c_{1} \rho V+c_{2} \int_{0}^{\infty} \bar{F}(z) d z-\left(c_{1}+c_{2}\right) \int_{0}^{\rho V} \bar{F}(z) d z\right] \\
=C_{\sigma}^{\bullet}(\rho, V)
\end{gathered}
$$

and

$$
\begin{aligned}
T_{\sigma}(\tau) & =\int_{0}^{\tau} \bar{F}\left(\frac{x}{\sigma}\right) d x=\sigma\left[\int_{0}^{\tau} \bar{F}\left(\frac{x}{\sigma}\right) d\left(\frac{x}{\sigma}\right)\right] \\
& =\sigma\left[\int_{0}^{\rho V} \bar{F}(z) d z\right]=T_{\sigma}^{\bullet}(\rho, V),
\end{aligned}
$$

where

$$
\rho=\frac{\tau}{S}
$$

is an ancillary factor. Using the pivotal quantity averaging approach, one can obtain

$$
\begin{gathered}
C_{\sigma}^{\bullet}(\rho)=E\left\{C_{\sigma}^{\bullet}(\rho, V)\right\} \\
=\sigma E\left\{c_{1} \rho V+c_{2} \int_{0}^{\infty} \bar{F}(z) d z-\left(c_{1}+c_{2}\right) \int_{0}^{\rho V} \bar{F}(z) d z\right\}, \\
T_{\sigma}^{\bullet}(\rho)=E\left\{T_{\sigma}^{\bullet}(\rho, V)\right\}=\sigma E\left\{\left[\int_{0}^{\rho V} \bar{F}(z) d z\right]\right\},
\end{gathered}
$$

and

$$
\begin{gathered}
=\frac{\Lambda^{\bullet}(\rho)=\frac{C_{\sigma}^{\bullet}(\rho)}{T_{\sigma}^{\bullet}(\rho)}}{\sigma E\left\{c_{1} \rho V+c_{2} \int_{0}^{\infty} \bar{F}(z) d z-\left(c_{1}+c_{2}\right) \int_{0}^{\rho V} \bar{F}(z) d z\right\}} \\
=\frac{\sigma E\left\{\int_{0}^{\rho V} \bar{F}(z) d z\right\}}{c_{1} \rho E\{V\}+c_{2} \int_{0}^{\infty} \bar{F}(z) d z} \\
E\left\{\int_{0}^{\rho V} \bar{F}(z) d z\right\}
\end{gathered}
$$

Thus, the unknown parameter $\sigma$ is eliminated from the problem. Now, an optimal statistical decision (based on pivotal quantity averaging) for a preventive replacement age is determined as

$$
\tau^{\mathrm{PQA}}=\rho^{\mathrm{PQA}} S
$$

where

$$
\begin{gathered}
\rho^{\mathrm{PQA}}=\underset{\rho}{\arg \min }\left(\Lambda^{\bullet}(\rho)\right) \\
=\arg \min _{\rho}\left(\frac{c_{1} \rho E\{V\}+c_{2} \int_{0}^{\infty} \bar{F}(z) d z}{E\left\{\int_{0}^{\rho V} \bar{F}(z) d z\right\}}-\left(c_{1}+c_{2}\right)\right) .
\end{gathered}
$$

Comparison of statistical decisions. For comparison, a maximum likelihood estimate $\tau^{\mathrm{ML}}$ of $\tau^{*}$ is determined as

$$
\tau^{\mathrm{ML}}=\arg \min _{\tau}\left[\Lambda_{\hat{\sigma}}(\tau)=\frac{C_{\hat{\sigma}}(\tau)}{T_{\hat{\sigma}}(\tau)}\right]
$$

$$
=\arg \min _{\tau}\left[\frac{c_{1} \tau+c_{2} E_{\hat{\sigma}}\{X\}-\left(c_{1}+c_{2}\right) \int_{0}^{\tau} \bar{F}_{\hat{\sigma}}(x) d x}{\int_{0}^{\tau} \bar{F}_{\hat{\sigma}}(x) d x}\right]
$$

$$
=\arg \min _{\tau}\left[\frac{c_{1} \tau+c_{2} E_{\hat{\sigma}}\{X\}}{\tau}-\left(c_{1}+c_{2}\right)\right]
$$




$$
=\arg \left(\tau=\left(\bar{F}_{\hat{\sigma}}(\tau)\right)^{-1} \int_{0}^{\tau} \bar{F}_{\hat{\sigma}}(x) d x-\frac{c_{2}}{c_{1}} E_{\hat{\sigma}}\{X\}\right),
$$

where $\hat{\sigma}$ is a maximum likelihood estimate of $\sigma$. In this case, the index of improvement percentage in effectiveness of a replacement policy of the pivotal quantity averaging approach as compared with the maximum likelihood approach is given by

$$
I_{\text {imp.per }}\left(\tau^{\mathrm{PQA}}, \tau^{\mathrm{ML}}\right)=\frac{\Lambda^{\bullet}\left(\rho^{\mathrm{ML}}\right)-\Lambda^{\bullet}\left(\rho^{\mathrm{PQA}}\right)}{\Lambda^{\bullet}\left(\rho^{\mathrm{ML}}\right)} 100 \%,
$$

where

$$
\rho^{\mathrm{ML}}=\frac{\tau^{\mathrm{ML}}}{S}
$$

\subsection{Location-Scale Family of Lifetime Distributions}

Consider a situation described by a location-scale family of probability distribution functions, indexed by a parametric vector $\theta=(\mu, \sigma)$,

$$
\begin{gathered}
F_{\theta}(x)=\operatorname{Pr}(X \leq x)=F\left(\frac{x-\mu}{\sigma}\right)=F(z), \\
-\infty<x<\infty
\end{gathered}
$$

where $-\infty<\mu<\infty$ is a location parameter and $\sigma>0$ is a scale parameter, the distribution of $Z=(X-\mu) / \sigma$ does not depend on any unknown parameters. Let $X_{1}, X_{2}, \ldots, X_{n}$ denote a random sample of component lifetimes, each possessing a distribution function (42) such that $E_{\theta}\{X\}$ is finite. It is assumed that the scale parameter is unknown, but there exists some statistic $S=$ $S\left(X_{1}, X_{2}, \ldots, X_{n}\right)=\left(S_{1}, S_{2}\right)$ such that

$$
V_{1}=\frac{S_{1}-\mu}{\sigma}, \quad V_{2}=\frac{S_{2}}{\sigma}
$$

represent pivotal quantities with probability distributions that do not depend on any unknown parameter. In this case, the expected cost per unit (7) and the expected usage per unit (8) are transformed, respectively, as follows:

$$
\begin{aligned}
C_{\theta}(\tau)=c_{1} \tau & +c_{2} \int_{-\infty}^{\infty} \bar{F}\left(\frac{x-\mu}{\sigma}\right) d x-\left(c_{1}+c_{2}\right) \int_{-\infty}^{\tau} \bar{F}\left(\frac{x-\mu}{\sigma}\right) d x \\
= & \sigma\left[c_{1} \frac{\tau}{\sigma}+c_{2} \int_{-\infty}^{\infty} \bar{F}\left(\frac{x-\mu}{\sigma}\right) d\left(\frac{x-\mu}{\sigma}\right)\right. \\
& \left.-\left(c_{1}+c_{2}\right) \int_{-\infty}^{\tau} \bar{F}\left(\frac{x-\mu}{\sigma}\right) d\left(\frac{x-\mu}{\sigma}\right)\right]
\end{aligned}
$$

$$
\begin{array}{r}
=\sigma\left[c_{1}\left(\rho+S_{1} / S_{2}\right) V_{2}+c_{2} \int_{-\infty}^{\infty} \bar{F}(z) d z\right. \\
\left.-\left(c_{1}+c_{2}\right) \int_{-\infty}^{\rho V_{2}+V_{1}} \bar{F}(z) d z\right]=C_{\sigma S}^{\bullet}\left(\rho, V_{1}, V_{2}\right)
\end{array}
$$

and

$$
\begin{aligned}
T_{\theta}(\tau) & =\int_{-\infty}^{\tau} \bar{F}\left(\frac{x}{\sigma}\right) d x=\sigma\left[\int_{-\infty}^{\tau} \bar{F}\left(\frac{x-\mu}{\sigma}\right) d\left(\frac{x-\mu}{\sigma}\right)\right] \\
& =\sigma\left[\int_{0}^{\rho V_{2}+V_{1}} \bar{F}(z) d z\right]=T_{\sigma}^{\bullet}\left(\rho, V_{1}, V_{2}\right),
\end{aligned}
$$

where

$$
\rho=\frac{\tau-S_{1}}{S_{2}}
$$

is an ancillary factor. Using the pivotal quantity averaging approach, one can obtain

$$
\begin{gathered}
C_{\sigma S}^{\bullet}(\rho)=E\left\{C_{\sigma S}^{\bullet}\left(\rho, V_{1}, V_{2}\right)\right\} \\
=\sigma E\left\{c_{1}\left(\rho+S_{1} / S_{2}\right) V_{2}+c_{2} \int_{-\infty}^{\infty} \bar{F}(z) d z\right. \\
\left.-\left(c_{1}+c_{2}\right) \int_{-\infty}^{\rho V_{2}+V_{1}} \bar{F}(z) d z\right\}, \\
T_{\sigma}^{\bullet}(\rho)=E\left\{T_{\sigma}^{\bullet}\left(\rho, V_{1}, V_{2}\right)\right\}=\sigma E\left\{\int_{-\infty}^{\rho V_{2}+V_{1}} \bar{F}(z) d z\right\},
\end{gathered}
$$

and

$$
\begin{gathered}
\Lambda_{S}^{\bullet}(\rho)=\frac{C_{\sigma S}^{\bullet}(\rho)}{T_{\sigma}^{\bullet}(\rho)} \\
=\frac{\sigma E\left\{c_{1}\left(\rho+S_{1} / S_{2}\right) V_{2}+c_{2} \int_{-\infty}^{\infty} \bar{F}(z) d z-\left(c_{1}+c_{2}\right) \int_{-\infty}^{\rho V_{2}+V_{1}} \bar{F}(z) d z\right\}}{\sigma E\left\{\int_{-\infty}^{\rho V_{2}+V_{1}} \bar{F}(z) d z\right\}} \\
=\frac{c_{1}\left(\rho+S_{1} / S_{2}\right) E\left\{V_{2}\right\}+c_{2} \int_{-\infty}^{\infty} \bar{F}(z) d z}{E\left\{\int_{-\infty}^{\rho V_{2}+V_{1}} \bar{F}(z) d z\right\}}-\left(c_{1}+c_{2}\right) .
\end{gathered}
$$


Thus, the unknown parametric vector $\theta$ is eliminated from the problem. Now, an optimal statistical decision (based on pivotal quantity averaging) for a preventive replacement age is determined as

$$
\tau^{\mathrm{PQA}}=S_{1}+\rho^{\mathrm{PQA}} S_{2}
$$

where

$$
\rho^{\mathrm{PQA}}=\underset{\rho}{\arg \min }\left(\Lambda_{S}^{\bullet}(\rho)\right)
$$

$=\arg \min _{\rho}\left(\frac{c_{1}\left(\rho+S_{1} / S_{2}\right) E\left\{V_{2}\right\}+c_{2} \int_{-\infty}^{\infty} \bar{F}(z) d z}{E\left\{\int_{-\infty}^{\rho V_{2}+V_{1}} \bar{F}(z) d z\right\}}-\left(c_{1}+c_{2}\right)\right)$.

Comparison of statistical decisions. For comparison, a maximum likelihood estimate $\tau^{\mathrm{ML}}$ of $\tau^{*}$ is determined as

$$
\begin{aligned}
& \tau^{\mathrm{ML}}=\arg \min _{\tau}\left(\Lambda_{\hat{\theta}}(\tau)=\frac{C_{\hat{\theta}}(\tau)}{T_{\hat{\theta}}(\tau)}\right) \\
& =\arg \min _{\tau}\left(\frac{c_{1} \tau+c_{2} E_{\hat{\theta}}\{X\}-\left(c_{1}+c_{2}\right) \int_{0}^{\tau} \bar{F}_{\hat{\theta}}(x) d x}{\int_{0}^{\tau} \bar{F}_{\hat{\theta}}(x) d x}\right) \\
& =\arg \min _{\tau}\left(\frac{c_{1} \tau+c_{2} E_{\hat{\theta}}\{X\}}{\int_{0}^{\tau} \bar{F}_{\hat{\theta}}(x) d x}-\left(c_{1}+c_{2}\right)\right) \\
& =\arg \left(\tau=\left(\bar{F}_{\hat{\theta}}(\tau)\right)^{-1} \int_{0}^{\tau} \bar{F}_{\hat{\theta}}(x) d x-\frac{c_{2}}{c_{1}} E_{\hat{\theta}}\{X\}\right),
\end{aligned}
$$

where $\hat{\theta}$ is a maximum likelihood estimate of $\theta$. In this case, the index of improvement percentage in effectiveness of a replacement policy of the pivotal quantity averaging approach as compared with the maximum likelihood approach is given by

$$
I_{\text {imp.per }}\left(\tau^{\mathrm{PQA}}, \tau^{\mathrm{ML}}\right)=\frac{\Lambda_{S}^{\bullet}\left(\rho^{\mathrm{ML}}\right)-\Lambda_{S}^{\bullet}\left(\rho^{\mathrm{PQA}}\right)}{\Lambda_{S}^{\bullet}\left(\rho^{\mathrm{ML}}\right)} 100 \% \text {, }
$$

where

$$
\rho^{\mathrm{ML}}=\frac{\tau^{\mathrm{ML}}-S_{1}}{S_{2}}
$$

\subsection{Numerical Example 2}

One-parameter exponential distribution of lifetime. Let us assume that a unit's lifetime is represented by a random quantity

$$
X \sim F_{\sigma}(x)=1-\exp \left(-\frac{x}{\sigma}\right)
$$

where the parameter $\sigma$ is unknown. The aim is to determine an optimal statistical decision for preventive replacement age $\tau$. Let $X_{1}, X_{2}, \ldots, X_{n}$ be a sample of independent and identically distributed random quantities from (55). It is known that the statistic

$$
S=\sum_{i=1}^{n} X_{i}
$$

is sufficient for $\sigma$ with the probability density function

$$
f_{\sigma}(s)=\frac{1}{\Gamma(n) \sigma^{n}} s^{n-1} \exp \left(-\frac{s}{\sigma}\right), \quad s>0
$$

where the pivotal quantity $V=S / \sigma$ has the probability density function

$$
f(v)=\frac{1}{\Gamma(n)} v^{n-1} \exp (-v), \quad v>0 .
$$

If $n=1, S=2000, \mathrm{c}_{1}=1000, \mathrm{c}_{2}=100$, it follows from (38) that

$$
\begin{gathered}
\rho^{\mathrm{PQA}}=\underset{\rho}{\arg \min }\left(\Lambda^{\bullet}(\rho)\right) \\
=\underset{\rho}{\arg \min }\left(\frac{c_{1} \rho E\{V\}+c_{2} \int_{0}^{\infty} \bar{F}(z) d z}{E\left\{\int_{0}^{\rho V} \bar{F}(z) d z\right\}}-\left(c_{1}+c_{2}\right)\right) \\
=\arg \min \left(\frac{c_{1} \rho n+c_{2}}{1-1 /(1+\rho)^{n}}-\left(c_{1}+c_{2}\right)\right)=0.316228, \\
\Lambda_{\left(\rho^{\mathrm{PQA}}\right)=}^{\left.\frac{c_{1} \rho^{\mathrm{PQA}}}{1-1 /\left(1+c_{2}\right.} \rho^{\mathrm{PQA}}\right)^{n}-\left(c_{1}+c_{2}\right)=632.4555,} \\
\tau^{\mathrm{PQA}}=\rho^{\mathrm{PQA}} S=632.4559 ; \\
\tau^{\mathrm{ML}}=\arg \min \left[\Lambda_{\hat{\sigma}}(\tau)\right]
\end{gathered}
$$




$$
\left.\begin{array}{c}
=\arg \min _{\tau}\left[\frac{c_{1} \tau+c_{2} E_{\hat{\sigma}}\{X\}}{\tau}-\left(c_{1}+c_{2}\right)\right]=832.4423, \\
\int_{0}^{\tau} \bar{F}_{\hat{\sigma}}(x) d x
\end{array}\right]
$$

where

$$
\rho^{\mathrm{ML}}=\frac{\tau^{\mathrm{ML}}}{S}=0.416221
$$

Comparison of statistical decisions. Thus, the index of improvement percentage in effectiveness of a replacement policy of the pivotal quantity averaging approach as compared with the maximum likelihood approach is given by

$$
\begin{gathered}
I_{\text {imp.per }}\left(\tau^{\mathrm{PQA}}, \tau^{\mathrm{ML}}\right) \\
=\frac{\Lambda^{\bullet}\left(\rho^{\mathrm{ML}}\right)-\Lambda^{\bullet}\left(\rho^{\mathrm{PQA}}\right)}{\Lambda^{\bullet}\left(\rho^{\mathrm{ML}}\right)} 100 \%=3.66 \%
\end{gathered}
$$

\section{Conclusion}

In this paper, an innovative age replacement model is proposed. It is suitable for situations where the costs included in the model are not assumed to be constants. For effective optimization of statistical decisions for age replacement problems under parametric uncertainty, the pivotal quantity averaging (PQA) approach is suggested. It represents a new simple and computationally attractive statistical technique based on the constructive use of the invariance principle in mathematical statistics. This technique allows one to eliminate unknown parameters from the problem and to find the better decision rules, which are more effective than any of the well-known decision rules. The methodology, which is suggested in this paper, may be found to be useful for the use in many industries with parametric uncertainty of underlying models to better manage the information that we already have. While the details of problems considered in the paper can change significantly from one industry to the next, the focus is always on making better statistical decisions and predictive inferences - and not manually with guess work and intuition but rather scientifically with models and technology, all implemented with disciplined processes and systems. Thus, the paper focuses on the development and implementation of new methodologies and technologies that should help one to effectively solve many real practical problems.

\section{References}

[1] R. E. Barlow and L. C. Hunter, "Optimal preventive maintenance policies," Operations Research, vol. 8, pp. 90-100, 1960 .

[2] R. E. Barlow and F. Proschan. Mathematical Theory of Reliability. New York: Wiley, 1965.

[3] R. E. Barlow and F. Proschan, Statistical Theory of Reliability and Life Testing: Probability Models. New York: Holt, Rinehart, and Winston, 1975.

[4] H. Ascher and H. Feingold, Repairable Systems Reliability: Modeling, Inference, Misconceptions and their Causes. New York: Marcel Dekker, Inc., 1984.

[5] T. Nakagawa, "Summary of periodic replacement with minimal repair at failure," Journal of the Operations Research Society of Japan, vol. 24, pp. 213-228, 1981.

[6] C. Valdez-Flares and R. M. Feldman, "A survey of preventive maintenance models for stochastically deteriorating single-unit systems," Naval Research Logistics, vol. 36, pp. 419-446, 1989.

[7] R. I. Phelps, “Optimal policy for minimal repair," Journal of the Operational Research Society, vol. 34, pp. 425-427, 1983.

[8] A. Tahara and T. Nishida, "Optimal replacement policy for minimal repair model," Journal of the Operations Research Society of Japan, vol. 18, pp. 113-124, 1975.

[9] G. J. Glasser, “The age replacement problem,” Technometrics, vol. 9, pp. 83-91, 1967.

[10] R. L. Scheaffer, "Optimum age replacement policies with an increasing cost factor," Technometrics, vol. 13, pp. 139-144, 1971.

[11] M. B. Berg, "A marginal cost analysis for preventive maintenance policies," European Journal of Operational Research, vol. 4, pp. 136-142, 1980.

[12] N. A. Nechval and E. K. Vasermanis, Improved Decisions in Statistics. Riga: Izglitibas soli, 2004. 